\title{
B field and squeezed states in Vacuum String Field Theory
}

\author{
L.Bonora, D.Mamone, M.Salizzoni \\ International School for Advanced Studies (SISSA/ISAS) \\ Via Beirut 2-4, 34014 Trieste, Italy, and INFN, Sezione di Trieste \\ E-mail: bonora@sissa.it, mamone@sissa.it, sali@sissa.it
}

\begin{abstract}
We show that squeezed state solutions for solitonic lumps in Vacuum String Field Theory still exist in the presence of a constant $B$ field. We show in particular that, just as in the $B=0$ case, we can write down a compact explicit form for such solutions.
\end{abstract}

Keywords: String Field Theory, Squeezed States, B field, Solitonic Lumps. 


\section{Contents}

1. Introduction

2. The three string vertex in the presence of a constant background B field 3

3. Properties of the new coefficients 6

4. The squeezed state solution 10

5. A comment 12

A. Derivation of $(\mathcal{U} \tilde{U})_{N M}^{\alpha \beta}$

\section{Introduction}

What happens in Witten's Open String Field Theory, [1], when a constant $B$ field is switched on? This question has already been partially answered. In ref. [4] and [5] it was shown that in the limit of field theory the string field theory $*$ product factorizes into the ordinary Witten $*$ product and the Moyal product. A related result can be obtained in the following way. The string field theory action

$$
\mathcal{S}(\Psi)=-\frac{1}{g_{0}^{2}}\left[\frac{1}{2}\langle\Psi, Q \Psi\rangle+\frac{1}{3}\langle\Psi, \Psi * \Psi\rangle\right]
$$

can be explicitly calculated in terms of local fields, provided the string field is expressed itself in terms of local fields

$$
|\Psi\rangle=\left(\phi(x)+A_{\mu}(x) a_{1}^{\mu \dagger}+\ldots\right) c_{1}|0\rangle
$$

Of course this makes sense in the limit in which string theory can be approximated by a local field theory. In this framework (1.1) takes the form of an integrated function $F$ of (an infinite series of) local polynomials (kinetic and potential terms) of the fields involved in (1.2):

$$
\mathcal{S}(\Psi)=\int d^{26} x F\left(\varphi_{i}, \partial \varphi_{i}, \ldots\right)
$$

Now, it has been proven by [2, 3] that, when a $B$ field is switched on, the kinetic term of (1.1) remains the same while the three string vertex changes, being multiplied by a (cyclically invariant) noncommutative phase factor (see ([2, 3]) and eq. 2.5) below). It is easy to see on a general basis that the overall effect of such noncommutative factor is to 
replace the ordinary product with the Moyal product in the RHS of the effective action (1.3) ${ }^{1}$.

Therefore, we know pretty well the effects of a $B$ field in the field theory limit of SFT. What we want to explore in this paper are the effects of a $B$ field in a nonperturbative regime. The good news that comes from the present investigation is that a thorough analysis of such effects can be carried out for squeezed states and exact solutions can be written down for tachyonic lumps.

In the context of the Vacuum String Field Theory (VSFT) squeezed states are very important solutions of the SFT equations of motion, [9]. There are strong arguments in favor of their interpretation as D-branes, [10, 11, 12], see also [15, 16, 22]. For a detailed definition of VSFT see [10]. The VSFT action has the same form as (1.1) with the BRST operator $Q$ replaced by a new one, usually denoted $\mathcal{Q}$, with the characteristic of being universal. As a matter of fact in [13], see also [14, 17, 18, 19, 20, 21] and [12, 23, 24, 25], an explicit representation of $\mathcal{Q}$ has been proposed, purely in terms of ghost fields. Now, the equation of motion of VSFT is

$$
\mathcal{Q} \Psi=-\Psi * \Psi
$$

and nonperturbative solutions are looked for in the form

$$
\Psi=\Psi_{m} \otimes \Psi_{g}
$$

where $\Psi_{g}$ and $\Psi_{m}$ depend purely on ghost and matter degrees of freedom, respectively. Then eq.(1.4) splits into

$$
\begin{aligned}
\mathcal{Q} \Psi_{g} & =-\Psi_{g} * \Psi_{g} \\
\Psi_{m} & =\Psi_{m} * \Psi_{m}
\end{aligned}
$$

Eq.(1.6) will not be involved in our analysis since ghosts are unaffected by the presence of a $B$ field. Therefore we will concentrate on the solutions of (1.7).

The value of the action for such solutions is given by

$$
\mathcal{S}(\Psi)=\mathcal{K}\left\langle\Psi_{m} \mid \Psi_{m}\right\rangle
$$

where $\mathcal{K}$ contains the ghost contribution. The point is that, as shown in [12], $\mathcal{K}$ is infinite unless it is suitably regularized. It has been argued in [12] that this should be understood as a 'gauge' freedom in choosing the solutions of (1.4), so that a coupled solution of (1.6) and (1.7), even if it is naively singular in its ghost component, is nevertheless a legitimate representative of the corresponding class of solutions.

\footnotetext{
${ }^{1}$ Actually in (过, 3]) it was suggested, more drastically, that by means of a string field redefinition one can reduce the modified SFT action to the original form (1.1). However it is not clear, at least to us, what are the allowed string field redefinitions in SFT. For instance in the field theory limit, we have just seen that the terms of the ordinary effective action are replaced by analogous terms with the ordinary product replaced by the Moyal product. But, for instance, there is no local field theory redefinition that allows us to pass from such a term as $\int \phi \star \phi \star \phi$, where $\star$ denotes the Moyal product, to $\int \phi(x)^{3}$. We therefore believe that a closer look at the effects of turning on a $B$ field is necessary.
} 
The aim of this paper is to find a solutions of eq.(1.7) when a constant $B$ field is switched on along some space directions. It is rewarding that not only such solutions exist in the form of squeezed states, but also that they may be cast in a simple compact form. We can phrase it shortly by saying that eq.(1.7) is 'exactly solvable' even in the presence of a constant $B$ field.

The paper is organized as follows. In the next section we write down explicitly the algebraic form of the three string vertex in the presence of a constant $B$ field. In section 3 we study the properties of the numerical coefficients (Neumann coefficients) of the new three string vertex. Finally in section 4 we find exact solutions of (1.7) in the form of squeezed states.

\section{The three string vertex in the presence of a constant background $B$ field}

The three string vertex [1, 0, 8] of the Open String Field Theory is given by

$$
\left|V_{3}\right\rangle=\int d^{26} p_{(1)} d^{26} p_{(2)} d^{26} p_{(3)} \delta^{26}\left(p_{(1)}+p_{(2)}+p_{(3)}\right) \exp (-E)|0, p\rangle_{123}
$$

where

$$
E=\sum_{r, s=1}^{3}\left(\frac{1}{2} \sum_{m, n \geq 1} \eta_{\mu \nu} a_{m}^{(r) \mu \dagger} V_{m n}^{r s} a_{n}^{(s) \nu \dagger}+\sum_{n \geq 1} \eta_{\mu \nu} p_{(r)}^{\mu} V_{0 n}^{r s} a_{n}^{(s) \nu \dagger}+\frac{1}{2} \eta_{\mu \nu} p_{(r)}^{\mu} V_{00}^{r s} p_{(s)}^{\nu}\right)
$$

Summation over the Lorentz indices $\mu, \nu=0, \ldots, 25$ is understood and $\eta$ denotes the flat Lorentz metric and the operators $a_{m}^{(r) \mu}, a_{m}^{(r) \mu \dagger}$ denote the non-zero modes matter oscillators of the $r$-th string, which satisfy

$$
\left[a_{m}^{(r) \mu}, a_{n}^{(s) \nu \dagger}\right]=\eta^{\mu \nu} \delta_{m n} \delta^{r s}, \quad m, n \geq 1
$$

$p_{(r)}$ is the momentum of the $r$-th string and $|0, p\rangle_{123} \equiv\left|p_{(1)}\right\rangle \otimes\left|p_{(2)}\right\rangle \otimes\left|p_{(3)}\right\rangle$ is the tensor product of the Fock vacuum states relative to the three strings. $\left|p_{(r)}\right\rangle$ is annihilated by the annihilation operators $a_{m}^{(r) \mu}$ and is eigenstate of the momentum operator $\hat{p}_{(r)}^{\mu}$ with eigenvalue $p_{(r)}^{\mu}$. The normalization is

$$
\left\langle p_{(r)} \mid p_{(s)}^{\prime}\right\rangle=\delta_{r s} \delta^{26}\left(p+p^{\prime}\right)
$$

The coefficients $V_{M N}^{r s}(M(N)$ denotes from now on the couple $\{0, m\}(\{0, n\}))$ have been computed in [7, 8. We will use them in the notation of Appendix A and B of [1].

Our first goal is to find the new form of the coefficients $V_{M N}^{r s}$ when a constant $B$ field is switched on. We start from the simplest case, i.e. when $B$ is nonvanishing in the two space directions, say the 24-th and 25-th ones. Let us denote these directions with the Lorentz indices $\alpha$ and $\beta$. Then, as is well-known [6, 2, 3], in these two direction we have a new effective metric $G_{\alpha \beta}$, the open string metric, as well as an effective antisymmetric parameter $\theta_{\alpha \beta}$, given by

$G^{\alpha \beta}=\left(\frac{1}{\eta+2 \pi \alpha^{\prime} B} \eta \frac{1}{\eta-2 \pi \alpha^{\prime} B}\right)^{\alpha \beta}, \quad \theta^{\alpha \beta}=-\left(2 \pi \alpha^{\prime}\right)^{2}\left(\frac{1}{\eta+2 \pi \alpha^{\prime} B} B \frac{1}{\eta-2 \pi \alpha^{\prime} B}\right)^{\alpha \beta}$ 
Henceforth we set $\alpha^{\prime}=1$. This is in order to conform to the convention of [11], whose results will be compared with ours.

The three string vertex is modified only in the 24-th and 25-th direction, which, in view of the subsequent $\mathrm{D}$-brane interpretation, we call the transverse directions. We split the three string vertex into the tensor product of the perpendicular part and the parallel part

$$
\left|V_{3}\right\rangle=\left|V_{3, \perp}\right\rangle \otimes\left|V_{3, \|}\right\rangle
$$

The parallel part is the same as in the ordinary case and will not be re-discussed here. On the contrary we will describe in detail the perpendicular part of the vertex. We rewrite the exponent $E$ as $E=E_{\|}+E_{\perp}$, according to the above splitting. $E_{\perp}$ will be modified as follows

$$
\begin{aligned}
E_{\perp} \rightarrow E_{\perp}^{\prime}= & \sum_{r, s=1}^{3}\left(\frac{1}{2} \sum_{m, n \geq 1} G_{\alpha \beta} a_{m}^{(r) \alpha \dagger} V_{m n}^{r s} a_{n}^{(s) \beta \dagger}+\sum_{n \geq 1} G_{\alpha \beta} p_{(r)}^{\alpha} V_{0 n}^{r s} a_{n}^{(s) \beta \dagger}\right. \\
& \left.+\frac{1}{2} G_{\alpha \beta} p_{(r)}^{\alpha} V_{00}^{r s} p_{(s)}^{\beta}+\frac{i}{2} \sum_{r<s} p_{\alpha}^{(r)} \theta^{\alpha \beta} p_{\beta}^{(s)}\right)
\end{aligned}
$$

Next, as far as the zero modes are concerned, we pass from the momentum to the oscillator basis, [0], 8]. We define

$$
a_{0}^{(r) \alpha}=\frac{1}{2} \sqrt{b} \hat{p}^{(r) \alpha}-i \frac{1}{\sqrt{b}} \hat{x}^{(r) \alpha}, \quad a_{0}^{(r) \alpha \dagger}=\frac{1}{2} \sqrt{b} \hat{p}^{(r) \alpha}+i \frac{1}{\sqrt{b}} \hat{x}^{(r) \alpha},
$$

where $\hat{p}^{(r) \alpha}, \hat{x}^{(r) \alpha}$ are the zero momentum and position operator of the $r$-th string, and we have kept the 'gauge' parameter $b$ of ref. [11] $\left(b \sim \alpha^{\prime}\right)$. It is understood that $p^{(r) \alpha}=G^{\alpha \beta} p_{\beta}^{(r)}$. We have

$$
\left[a_{0}^{(r) \alpha}, a_{0}^{(s) \beta \dagger}\right]=G^{\alpha \beta} \delta^{r s}
$$

Denoting by $\left|\Omega_{b, \theta}\right\rangle$ the oscillator vacuum $\left(a_{0}^{\alpha}\left|\Omega_{b, \theta}\right\rangle=0\right)$, the relation between the momentum basis and the oscillator basis is defined by

$$
\begin{aligned}
& \left|p^{24}\right\rangle_{123} \otimes\left|p^{25}\right\rangle_{123} \equiv\left|\left\{p^{\alpha}\right\}\right\rangle_{123}= \\
& \left(\frac{b}{2 \pi \sqrt{\operatorname{det} G}}\right)^{\frac{3}{2}} \exp \left[\sum_{r=1}^{3}\left(-\frac{b}{4} p_{\alpha}^{(r)} G^{\alpha \beta} p_{\beta}^{(r)}+\sqrt{b} a_{0}^{(r) \alpha \dagger} p_{\alpha}^{(r)}-\frac{1}{2} a_{0}^{(r) \alpha \dagger} G_{\alpha \beta} a_{0}^{(r) \beta \dagger}\right)\right]\left|\Omega_{b, \theta}\right\rangle
\end{aligned}
$$

Now we insert this equation inside $E_{\perp}^{\prime}$ and try to eliminate the momenta along the perpendicular directions by integrating them out. To this end we rewrite $E_{\perp}^{\prime}$ in the following way and, for simplicity, drop all the labels $\alpha, \beta$ and $r, s$ :

$$
E_{\perp}^{\prime}=\frac{1}{2} \sum_{m, n \geq 1} a_{m}^{\dagger} G V_{m n} a_{n}^{\dagger}+\sum_{n \geq 1} p V_{0 n} a_{n}^{\dagger}+\frac{1}{2} p\left[G^{-1}\left(V_{00}+\frac{b}{2}\right)+\frac{i}{2} \theta \epsilon \chi\right] p-\sqrt{b} p a_{0}^{\dagger}+\frac{1}{2} a_{0}^{\dagger} G a_{0}^{\dagger}
$$


where we have set $\theta^{\alpha \beta}=\epsilon^{\alpha \beta} \theta$ and introduced the matrices $\epsilon$ with entries $\epsilon^{\alpha \beta}$ and $\chi$ with entries

$$
\chi^{r s}=\left(\begin{array}{ccc}
0 & 1 & -1 \\
-1 & 0 & 1 \\
1 & -1 & 0
\end{array}\right)
$$

At this point we impose momentum conservation. There are three distinct ways to do that and eventually one has to (multiplicatively) symmetrize with respect to them. Let us start by setting $p_{3}=-p_{1}-p_{2}$ in $E_{\perp}^{\prime}$ and obtain an expression of the form

$$
p X_{00} p+\sum_{N \geq 0} p Y_{0 N} a_{N}^{\dagger}+\sum_{M, N \geq 0} a_{M}^{\dagger} Z_{M N} a_{N}^{\dagger}
$$

where, in particular, $X_{00}$ is given by

$$
X_{00}^{\alpha \beta, r s}=G^{\alpha \beta}\left(V_{00}+\frac{b}{2}\right) \eta^{r s}+i \frac{\theta}{4} \epsilon^{\alpha \beta} \epsilon^{r s}
$$

Here the indices $r, s$ take only the values 1,2 , and

$$
\eta=\left(\begin{array}{cc}
1 & 1 / 2 \\
1 / 2 & 1
\end{array}\right), \quad \epsilon=\left(\begin{array}{cc}
0 & 1 \\
-1 & 0
\end{array}\right)
$$

Now, as usual, we redefine $p$ so as eliminate the linear term in (2.9). At this point we can easily perform the Gaussian integration over $p_{(1)}, p_{(2)}$, while the remnant of (2.9) will be expressed in terms of the inverse of $X_{00}$ :

$$
\left(X_{00}^{-1}\right)^{\alpha \beta, r s}=\frac{2 A^{-1}}{4 a^{2}+3}\left(\frac{3}{2} G^{\alpha \beta}\left(\eta^{-1}\right)^{r s}-2 i a \hat{\epsilon}^{\alpha \beta} \epsilon^{r s}\right)
$$

where

$$
A=V_{00}+\frac{b}{2}, \quad a=\frac{\theta}{4 A} \sqrt{\operatorname{Det} G}, \quad \epsilon^{\alpha \beta}=\sqrt{\operatorname{Det} G} \hat{\epsilon}^{\alpha \beta}
$$

Let us use henceforth for the $B$ field the explicit form

$$
B_{\alpha \beta}=\left(\begin{array}{cc}
0 & B \\
-B & 0
\end{array}\right)
$$

so that

$$
\operatorname{DetG}=\left(1+(2 \pi B)^{2}\right)^{2}, \quad \theta \sqrt{\operatorname{DetG}}=-(2 \pi)^{2} B, \quad a=-\frac{\pi^{2}}{A} B
$$

Now one has to symmetrize with respect to the three possibilities of imposing the momentum conservation. Remembering the factors due to integration over the momenta and collecting the results one gets for the three string vertex in the presence of a $B$ field

$$
\left|V_{3}\right\rangle^{\prime}=\left|V_{3, \perp}\right\rangle^{\prime} \otimes\left|V_{3, \|}\right\rangle
$$

$\left|V_{3, \|}\right\rangle$ is the same as in the ordinary case (without $B$ field), while

$$
\left|V_{3, \perp}\right\rangle^{\prime}=K_{2} e^{-E^{\prime}}|\tilde{0}\rangle
$$


with

$$
\begin{aligned}
K_{2} & =\frac{\sqrt{2 \pi b^{3}}}{A^{2}\left(4 a^{2}+3\right)}(\operatorname{Det} G)^{1 / 4}, \\
E^{\prime} & =\frac{1}{2} \sum_{r, s=1}^{3} \sum_{M, N \geq 0} a_{M}^{(r) \alpha \dagger} \nu_{\alpha \beta, M N}^{r s} a_{N}^{(s) \beta \dagger}
\end{aligned}
$$

and $|\tilde{0}\rangle=|0\rangle \otimes\left|\Omega_{b, \theta}\right\rangle$. The coefficients $\mathcal{V}_{M N}^{\alpha \beta, r s}$ are given by

$$
\begin{aligned}
& \mathcal{V}_{00}^{\alpha \beta, r s}=G^{\alpha \beta} \delta^{r s}-\frac{2 A^{-1} b}{4 a^{2}+3}\left(G^{\alpha \beta} \phi^{r s}-i a \hat{\epsilon}^{\alpha \beta} \chi^{r s}\right) \\
& \mathcal{V}_{0 n}^{\alpha \beta, r s}=\frac{2 A^{-1} \sqrt{b}}{4 a^{2}+3} \sum_{t=1}^{3}\left(G^{\alpha \beta} \phi^{r t}-i a \hat{\epsilon}^{\alpha \beta} \chi^{r t}\right) V_{0 n}^{t s} \\
& \mathcal{V}_{m n}^{\alpha \beta, r s}=G^{\alpha \beta} V_{m n}^{r s}-\frac{2 A^{-1}}{4 a^{2}+3} \sum_{t, v=1}^{3} V_{m 0}^{r v}\left(G^{\alpha \beta} \phi^{v t}-i a \hat{\epsilon}^{\alpha \beta} \chi^{v t}\right) V_{0 n}^{t s}
\end{aligned}
$$

where, by definition, $V_{0 n}^{r s}=V_{n 0}^{s r}$, and

$$
\phi=\left(\begin{array}{ccc}
1 & -1 / 2 & -1 / 2 \\
-1 / 2 & 1 & -1 / 2 \\
-1 / 2 & -1 / 2 & 1
\end{array}\right)
$$

while the matrix $\chi$ has been defined above (2.8). These two matrices satisfy the algebra

$$
\chi^{2}=-2 \phi, \quad \phi \chi=\chi \phi=\frac{3}{2} \chi, \quad \phi^{2}=\frac{3}{2} \phi
$$

To end this section we would like to notice that the above results can be easily extended to the case in which the transverse directions are more than two (i.e. the 24-th and 25-th ones) and even. The canonical form of the transverse $B$ field is

$$
B_{\alpha \beta}=\left(\begin{array}{ccccc}
0 & B_{1} & & & \ldots \\
-B_{1} & 0 & & & \\
0 & & 0 & B_{2} & \\
\ldots & & -B_{2} & 0 & \ldots
\end{array}\right)
$$

It is not hard to see that each couple of conjugate transverse directions under this decomposition, can be treated in a completely independent way. The result is that each couple of directions $(26-i, 25-i)$, corresponding to the eigenvalue $B_{i}$, will be characterized by the same formulas (2.20, 2.21, 2.22) above with $B$ replaced by $B_{i}$.

\section{Properties of the new coefficients}

In this section we derive the properties of the coefficients $\mathcal{V}_{M N}^{\alpha \beta, r s}$ which are essential for the later developments. These properties are parallel to those enjoyed by the ordinary coefficients, [月, 8, 9, 11].

Let us quote first two straightforward properties of $\mathcal{V}_{M N}^{\alpha \beta, r s}$ : 
- (i) they are symmetric under the simultaneous exchange of all the three couples of indices;

- (ii) they are endowed with the property of cyclicity in the $r, s$ indices, i.e. $\mathcal{V}^{r s}=$ $v^{r+1, s+1}$, where $r, s=4$ is identified with $r, s=1$ and we have dropped the other indices.

The first property is immediate. The second can also be proven directly from eqs. (2.22). However, since it will be an easy consequence of eq.(3.11) below, we pass immediately to the derivation of the latter.

To this end we need the following representation of the coefficients $V_{0 n}^{r s}$, derived from [7]:

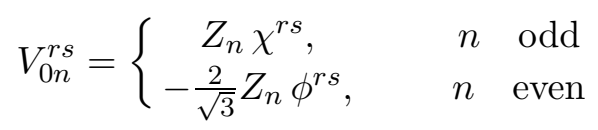

where

$$
Z_{n}=\sqrt{\frac{2}{3 n}} B_{0} A_{n}
$$

The numbers $B_{0}$ and $A_{n}$ were defined in ref.[0]. Notice that, since we have assumed $Z_{n}^{r s}=Z_{n}^{s r}$, we must have, by definition, $V_{0 n}^{r s}=V_{n 0}^{r s}$ for $n$ even and $V_{0 n}^{r s}=-V_{n 0}^{r s}$ for $n$ odd. Finally, for convenience, we introduce $Z_{0}=\sqrt{\frac{b}{3}}$.

Substituting (3.1) into eqs. 2.22) and using (2.24), we obtain

$$
\mathcal{V}_{N M}^{\alpha \beta, r s}=\left\{\begin{array}{ccc}
\mathcal{V}_{N M}^{\alpha \beta, r s}(\infty)-\frac{6 A^{-1}}{4 a^{2}+3} K_{\infty}^{\alpha \beta, r s} Z_{N} Z_{M}, & N+M & \text { even } \\
\mathcal{V}_{N M}^{\alpha \beta, r s}(\infty)+\frac{\sqrt{3} A^{-1}}{4 a^{2}+3} H_{\infty}^{\alpha \beta, r s}(-1)^{N} Z_{N} Z_{M}, & N+M & \text { odd }
\end{array}\right.
$$

In these equations

$$
\begin{aligned}
& K_{\infty}^{\alpha \beta, r s}=G^{\alpha \beta} \phi^{r s}-i a \hat{\epsilon}^{\alpha \beta} \chi^{r s} \\
& H_{\infty}^{\alpha \beta, r s}=3 G^{\alpha \beta} \chi^{r s}+4 i a \hat{\epsilon}^{\alpha \beta} \phi^{r s}
\end{aligned}
$$

and $\mathcal{V}_{N M}^{\alpha \beta, r s}(\infty)$ is

$$
\begin{aligned}
& \mathcal{V}_{00}^{\alpha \beta, r s}(\infty)=G^{\alpha \beta} \delta^{r s} \\
& \mathcal{V}_{0 m}^{\alpha \beta, r s}(\infty)=0 \\
& \mathcal{V}_{n m}^{\alpha \beta, r s}(\infty)=G^{\alpha \beta} V_{n m}^{r s}
\end{aligned}
$$

The coefficients $V_{n m}^{r s}$ are the same as in ref. [11] for $n, m \geq 1$.

We can also express the $\mathcal{V}_{N M}^{\alpha \beta, r s}$ in the following way

$$
\mathcal{V}_{N M}^{\alpha \beta, r s}=\left\{\begin{array}{ccc}
\mathcal{V}_{N M}^{\alpha \beta, r s}(0)+\frac{6 A^{-1}}{4 a^{2}+3} K_{0}^{\alpha \beta, r s} Z_{N} Z_{M}, & N+M & \text { even } \\
\mathcal{V}_{N M}^{\alpha \beta, r s}(0)+\frac{\sqrt{3} A^{-1}}{4 a^{2}+3} H_{0}^{\alpha \beta, r s}(-1)^{N} Z_{N} Z_{M}, & N+M & \text { odd }
\end{array}\right.
$$

where

$$
\begin{aligned}
& K_{0}^{\alpha \beta, r s}=\frac{4}{3} a^{2} G^{\alpha \beta} \phi^{r s}+i a \hat{\epsilon}^{\alpha \beta} \chi^{r s} \\
& H_{0}^{\alpha \beta, r s}=-4 a^{2} G^{\alpha \beta} \chi^{r s}+4 i a \hat{\epsilon}^{\alpha \beta} \phi^{r s}
\end{aligned}
$$


and $\mathcal{V}_{N M}^{\alpha \beta, r s}(0)=G^{\alpha \beta} V_{N M}^{\prime r s}$ are the values taken by $\mathcal{V}_{N M}^{\alpha \beta, r s}$ for $B=0$. As expected, the symbols $V_{N M}^{\prime r s}$ are the same as the coefficients $V_{n m}^{\prime r s}(b)$ with $n, m \geq 0$, used in [11].

Next we introduce the third root of unity $\omega=e^{i \frac{2 \pi}{3}}$ and notice that

$$
\phi^{r s}=\frac{1}{2}\left(\omega^{r-s}+\omega^{s-r}\right), \quad \chi^{r s}=\frac{i}{\sqrt{3}}\left(\omega^{r-s}-\omega^{s-r}\right),
$$

Inserting these relations into $(3.3,3.7)$ and rearranging the terms we find the basic relation

$$
\mathcal{V}_{N M}^{\alpha \beta, r s}=\frac{1}{3}\left(C_{N M}^{\prime} G^{\alpha \beta}+\omega^{s-r} \mathcal{U}_{N M}^{\alpha \beta}+\omega^{r-s} \overline{\mathcal{U}}_{N M}^{\alpha \beta}\right)
$$

where

$$
\mathcal{U}_{N M}^{\alpha \beta}=\left\{\begin{array}{ccc}
G^{\alpha \beta} \mathfrak{U}_{N M}(\infty)+R^{\alpha \beta} Z_{N} Z_{M}, & N+M & \text { even } \\
G^{\alpha \beta} \mathfrak{U}_{N M}(\infty)+i R^{\alpha \beta}(-1)^{N} Z_{N} Z_{M}, & N+M & \text { odd }
\end{array}\right.
$$

Moreover

$$
\bar{U}^{\alpha \beta}=\left(\mathcal{U}^{\beta \alpha}\right)^{*}
$$

where $^{*}$ denotes complex conjugation. In (3.11) $C_{N M}^{\prime}=(-1)^{N} \delta_{N M}$ and

$$
R^{\alpha \beta}=\frac{6 A^{-1}}{4 a^{2}+3}\left(-\frac{3}{2} G^{\alpha \beta}+\sqrt{3} a \hat{\epsilon}^{\alpha \beta}\right)
$$

Moreover

$$
\begin{aligned}
& \mathcal{U}_{00}^{\alpha \beta}(\infty)=G^{\alpha \beta}, \quad \mathcal{U}_{0 n}^{\alpha \beta}=0 \\
& \mathcal{U}_{n m}^{\alpha \beta}(\infty)=G^{\alpha \beta} U_{n m}
\end{aligned}
$$

In the last equation $U_{n m}$ coincides with the same symbol used in 11 (see eq.(B.15) in that reference).

Alternatively one can split $\mathcal{U}$ into the $B=0$ part and the rest. Then

$$
\mathcal{U}_{N M}^{\alpha \beta}=\left\{\begin{array}{ccc}
G^{\alpha \beta} \mathfrak{U}_{N M}(0)+T^{\alpha \beta} Z_{N} Z_{M}, & N+M & \text { even } \\
G^{\alpha \beta} \mathcal{U}_{N M}(0)+i T^{\alpha \beta}(-1)^{N} Z_{N} Z_{M}, & N+M & \text { odd }
\end{array}\right.
$$

where

$$
T^{\alpha \beta}=\frac{12 A^{-1}}{4 a^{2}+3}\left(a^{2} G^{\alpha \beta}+\frac{\sqrt{3}}{2} a \hat{\epsilon}^{\alpha \beta}\right)
$$

and $\mathcal{U}_{N M}^{\alpha \beta}=G^{\alpha \beta} U_{N M}^{\prime}$. The coefficients $U_{n m}^{\prime}, U_{0 n}^{\prime}, U_{00}^{\prime}$ are the same as in ref. 11] (see eq.(B.19) therein).

Let us discuss the properties of $\mathcal{U}$. Since

$$
\left(\mathcal{U}_{N M}^{\alpha \beta}\right)^{*}=\left\{\begin{array}{ccc}
\mathcal{U}_{N M}^{\alpha \beta}, & N+M & \text { even } \\
-\mathcal{U}_{N M}^{\alpha \beta}, & N+M & \text { odd }
\end{array}\right.
$$


it is easy to prove the following properties (where we use the matrix notation for the indices $N, M)$

$$
\left(\mathcal{U}^{\alpha \beta}\right)^{*}=C^{\prime} \mathfrak{U}^{\alpha \beta} C^{\prime}
$$

and

$$
\left(\mathcal{U}^{\alpha \beta}\right)^{\dagger}=\left(\mathcal{U}^{\alpha \beta}\right)^{* T}=\left(C^{\prime} \mathcal{U}^{\alpha \beta} C^{\prime}\right)^{T}=\mathcal{U}^{\alpha \beta}
$$

Finally, if tilde denotes transposition in the indices $\alpha, \beta$, it is possible to prove that (the proof is rather technical and deferred to Appendix A)

$$
(\mathcal{U} \tilde{U})_{N M}^{\alpha \beta}=(\tilde{\mathcal{U}})_{N M}^{\alpha \beta}=G^{\alpha \beta} \delta_{N M}+\left(R G+G \tilde{R}+\frac{2}{3} A R \tilde{R}\right) Z_{N} Z_{M}
$$

Now, remembering that $\hat{\epsilon}^{\alpha \gamma} \hat{\epsilon}_{\gamma}^{\beta}=-G^{\alpha \beta}$, it is elementary to prove that

$$
R G+G \tilde{R}+\frac{2}{3} A R \tilde{R}=0
$$

Therefore, finally,

$$
(\mathcal{U} \tilde{U})_{N M}^{\alpha \beta}=(\tilde{\mathcal{U}})_{N M}^{\alpha \beta}=G^{\alpha \beta} \delta_{N M}
$$

Eqs. 3.18, 3.19, 3.22) are the generalization of the analogous ones in [7, 8, 9, 11]. Using in particular (3.22), it is easy to prove that

$$
\left[C^{\prime} \mathcal{V}^{r s}, C^{\prime} \mathcal{V}^{r^{\prime} s^{\prime}}\right]=0
$$

This follows from

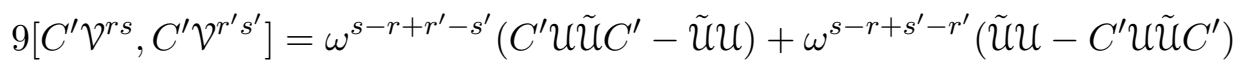

and from eq.(3.22). In the two previous equations matrix multiplication is understood both in the indices $M, N$ and $\alpha, \beta$. In the same sense, on the wake of 9, 11], we can also write down the following identities

$$
\begin{aligned}
& C^{\prime} \mathcal{V}^{12} C^{\prime} \mathcal{V}^{21}=C^{\prime} \mathcal{V}^{21} C^{\prime} \mathcal{V}^{12}=\left(C^{\prime} \mathcal{V}^{11}\right)^{2}-C^{\prime} \mathcal{V}^{11} \\
& \left(C^{\prime} \mathcal{V}^{12}\right)^{3}+\left(C^{\prime} \mathcal{V}^{21}\right)^{3}=2\left(C^{\prime} \mathcal{V}^{11}\right)^{3}-3\left(C^{\prime} \mathcal{V}^{11}\right)^{2}+G
\end{aligned}
$$

which will be needed in the next section.

Notice however that, unlike refs. [7, 8, 9, 11], we have

$$
C^{\prime} \mathcal{V}^{r s}=\tilde{\mathcal{V}}^{s r} C^{\prime}
$$




\section{The squeezed state solution}

A squeezed state in the present context is written as

$$
|S\rangle=\left|S_{\perp}\right\rangle \otimes\left|S_{\|}\right\rangle
$$

where $\left|S_{\|}\right\rangle$has the ordinary form, see [9, 11], and is treated in the usual way, while

$$
\begin{aligned}
& \left\langle S_{\perp}\right|=\mathcal{N}^{2}\langle\tilde{0}| \exp \left(-\frac{1}{2} \sum_{M, N \geq 0} a_{M}^{\alpha} \tilde{\mathcal{S}}_{\alpha \beta, M N} a_{N}^{\beta}\right) \\
& \left|S_{\perp}\right\rangle=\mathcal{N}^{2} \exp \left(-\frac{1}{2} \sum_{M, N \geq 0} a_{M}^{\alpha \dagger} \mathcal{S}_{\alpha \beta, M N} a_{N}^{\beta \dagger}\right)|\tilde{0}\rangle
\end{aligned}
$$

where $|\tilde{0}\rangle=\left|\Omega_{b, \theta}\right\rangle \otimes|0\rangle$. Here we have written down both bra and ket in order to stress the difference with [7, 8, 9, 11], which stems from the fact that, in view of (3.26), we assume $C^{\prime} \mathcal{S}^{\alpha \beta} C^{\prime}=\left(\mathcal{S}^{\alpha \beta}\right)^{*}=\mathcal{S}^{\beta \alpha}$. The $*$ product of two such states, labeled ${ }_{1}$ and ${ }_{2}$, is carried out in the same way as in the ordinary case, see again [9, 11]. Therefore we limit ourselves to writing down the result

$$
\left|S_{\perp}^{\prime}\right\rangle=\left|S_{1, \perp}\right\rangle *\left|S_{2, \perp}\right\rangle=\frac{K_{2}\left(\mathcal{N}_{1} \mathcal{N}_{2}\right)^{2}}{\operatorname{DET}(\mathbf{I}-\Sigma \mathcal{V})^{1 / 2}} \exp \left(-\frac{1}{2} \sum_{M, N \geq 0} a_{M}^{\alpha \dagger} \mathcal{S}_{\alpha \beta, M N}^{\prime} a_{N}^{\beta \dagger}\right)|\tilde{0}\rangle
$$

where, in matrix notation which includes both the indices $N, M$ and $\alpha, \beta$,

$$
\mathcal{S}^{\prime}=\mathcal{V}^{11}+\left(\mathcal{V}^{12}, \mathcal{V}^{21}\right)(\mathbf{I}-\Sigma \mathcal{V})^{-1} \Sigma\left(\begin{array}{l}
\mathcal{V}^{21} \\
\mathcal{V}^{12}
\end{array}\right)
$$

In RHS of these equations

$$
\Sigma=\left(\begin{array}{cc}
\tilde{\mathcal{S}}_{1} & 0 \\
0 & \tilde{\mathcal{S}}_{2}
\end{array}\right), \quad \mathcal{V}=\left(\begin{array}{cc}
\mathcal{V}^{11} & \mathcal{V}^{12} \\
\mathcal{V}^{21} & \mathcal{V}^{22}
\end{array}\right)
$$

and $\mathbf{I}_{\beta, M N}^{\alpha, r s}=\delta_{\beta}^{\alpha} \delta_{M N} \delta^{r s}, r, s=1,2$. DET is the determinant with respect to all indices. To reach the form (4.5) one has to use cyclicity of $\mathcal{V}^{r s}$ (property (ii) above). The expression of $\mathcal{S}^{\prime}$ is in fact a series, therefore some kind of condition on the coefficients $\mathcal{S}_{i}$ must be satisfied in order for it to make sense. The squeezed states $\mathcal{S}$ satisfying this condition form a subalgebra of the algebra defined by the $*$ product.

Let us now discuss the squeezed state solution of the equation $|\Psi\rangle *|\Psi\rangle=|\Psi\rangle$ in the matter sector. In order for this to be satisfied with the above states $|S\rangle$, we must first impose

$$
\mathcal{S}_{1}=\mathcal{S}_{2}=\mathcal{S}^{\prime} \equiv \mathcal{S}
$$

and then suitably normalize the resulting state. Then (4.5) becomes an equation for $\mathcal{S}$, i.e.

$$
\tilde{\mathcal{S}}=\mathcal{V}^{11}+\left(\mathcal{V}^{12}, \mathcal{V}^{21}\right)(\mathbf{I}-\Sigma \mathcal{V})^{-1} \Sigma\left(\begin{array}{l}
\mathcal{V}^{21} \\
\mathcal{V}^{12}
\end{array}\right)
$$


where $\Sigma, \mathcal{V}$ are the same as above with $\mathcal{S}_{1}=\mathcal{S}_{2}=\mathcal{S}$. Eq.4.7) has an obvious (formal) solution by iteration. However in ref. [9] it was shown that it is possible to obtain the solution in compact form by 'abelianizing' the problem. Notwithstanding the differences with that case, it is possible to reproduce the same trick on eq.(4.7), thanks to (3.23). One denotes $C^{\prime} \mathcal{V}^{r s}$ by $X^{r s}$ and $C^{\prime} \mathcal{S}$ by $\mathcal{T}$, and assumes that $\left[X^{r s}, \mathcal{T}\right]=0$ (of course this has to be checked a posteriori). Notice however that we cannot assume that $C^{\prime}$ commutes with $\mathcal{S}$, but we assume that $C^{\prime} \mathcal{S}=\tilde{S} C^{\prime}$. By multiplying (4.7) from the left by $C^{\prime}$ we get:

$$
\mathcal{T}=X^{11}+\left(X^{12}, X^{21}\right)(\mathbf{I}-\Sigma \mathcal{V})^{-1}\left(\begin{array}{c}
\mathcal{T} X^{21} \\
\mathcal{T} X^{12}
\end{array}\right)
$$

For instance $\tilde{\mathcal{S}} \mathcal{V}^{12}=\tilde{\mathcal{S}} C^{\prime} C^{\prime} \mathcal{V}^{12}=\mathcal{T} \mathcal{X}^{12}$, etc. In the same way,

$$
(\mathbf{I}-\Sigma \mathcal{V})^{-1}=\left(\begin{array}{cc}
\mathbb{I}-\mathcal{T} X^{11} & -\mathcal{T} X^{12} \\
-\mathcal{T} X^{21} & \mathbb{I}-\mathcal{T} \mathcal{X}^{11}
\end{array}\right)^{-1}
$$

where $\mathbb{I}_{\beta, M N}^{\alpha}=\delta_{\beta}^{\alpha} \delta_{M N}$. Now all the entries are commuting matrices, so the inverse can be calculated straight away.

From now on everything is the same as in [9, 11], therefore we limit ourselves to a quick exposition. Using (3.24) and (3.25), one arrives at an equation only in terms of $\mathcal{T}$ and $X \equiv X^{11}$ :

$$
(\mathcal{T}-\mathbb{I})\left(X \mathcal{T}^{2}-(\mathbb{I}+X) \mathcal{T}+X\right)=0
$$

This gives two solutions:

$$
\begin{aligned}
& \mathcal{T}=\mathbb{I} \\
& \mathcal{T}=\frac{1}{2 X}(\mathbb{I}+X-\sqrt{(\mathbb{I}+3 X)(\mathbb{I}-X)})
\end{aligned}
$$

The third solution, with a + sign in front of the square root, is not acceptable, as explained in [11]. In both cases we see that the solution commutes with $X^{r s}$. Naturally we are talking about solutions of the abelianized eq.(4.8). The true solution we are looking for is, in both cases, $\mathcal{S}=C^{\prime} \mathcal{T}$.

As for (4.10), it is easy to see that it leads to the identity state. Therefore, from now on we will consider (4.11) alone.

Now, let us deal with the normalization of $\left|S_{\perp}\right\rangle$. Imposing $\left|S_{\perp}\right\rangle *\left|S_{\perp}\right\rangle=\left|S_{\perp}\right\rangle$ we find

$$
\mathcal{N}^{2}=K_{2}^{-1} \operatorname{DET}(\mathbf{I}-\Sigma \mathcal{V})^{1 / 2}
$$

Replacing in it the solution one finds

$$
\operatorname{DET}(\mathbf{I}-\Sigma \mathcal{V})=\operatorname{Det}((\mathbb{I}-X)(\mathbb{I}+\mathcal{T}))
$$

Det denotes the determinant with respect to the indices $\alpha, \beta, M, N$. Using this equation and (2.18), and borrowing from [11] the expression for $\left|S_{\|}\right\rangle$, one finally gets for the 23dimensional tachyonic lump:

$$
|S\rangle=\left\{\operatorname{det}(1-X)^{1 / 2} \operatorname{det}(1+T)^{1 / 2}\right\}^{24} \exp \left(-\frac{1}{2} \eta_{\bar{\mu} \bar{\nu}} \sum_{m, n \geq 1} a_{m}^{\bar{\mu} \dagger} S_{m n} a_{n}^{\bar{\nu} \dagger}\right)|0\rangle \otimes
$$




$$
\frac{A^{2}\left(3+4 a^{2}\right)}{\sqrt{2 \pi b^{3}}(\operatorname{Det} G)^{1 / 4}}\left(\operatorname{Det}(\mathbb{I}-\mathcal{X})^{1 / 2} \operatorname{Det}(\mathbb{I}+\mathcal{T})^{1 / 2}\right) \exp \left(-\frac{1}{2} \sum_{M, N \geq 0} a_{M}^{\alpha \dagger} \mathcal{S}_{\alpha \beta, M N} a_{N}^{\beta \dagger}\right)|\tilde{0}\rangle
$$

where $\mathcal{S}=C^{\prime} \mathcal{T}$ and $\mathcal{T}$ is given by (4.11). The quantities in the first line are defined in ref. [11] with $\bar{\mu}, \bar{\nu}=0, \ldots 23$ denoting the parallel directions to the lump.

The value of the action corresponding to $(4.13)$ is easily calculated

$$
\begin{aligned}
\mathcal{S}_{\mathcal{S}}= & \mathcal{K} \frac{V^{(24)}}{(2 \pi)^{24}}\left\{\operatorname{det}(1-X)^{3 / 4} \operatorname{det}(1+3 X)^{1 / 4}\right\}^{24} \\
& \cdot \frac{A^{4}\left(3+4 a^{2}\right)^{2}}{2 \pi b^{3}(\operatorname{Det} G)^{1 / 2}} \operatorname{Det}(\mathbb{I}-X)^{3 / 4} \operatorname{Det}(\mathbb{I}+3 X)^{1 / 4}
\end{aligned}
$$

where $V^{(24)}$ is the volume along the parallel directions and $\mathcal{K}$ is the constant of eq.(1.8).

Finally, let $\mathfrak{e}$ denote the energy per unit volume, which coincides with the brane tension when $B=0$. Then one can compute the ratio of the D23-brane energy density $\mathfrak{e}_{23}$ to the D25-brane energy density $\mathfrak{e}_{25}$;

$$
\begin{aligned}
\frac{\mathfrak{e}_{23}}{\mathfrak{e}_{25}} & =\frac{(2 \pi)^{2}}{(\operatorname{Det} G)^{1 / 4}} \cdot \mathcal{R} \\
\mathcal{R} & =\frac{A^{4}\left(3+4 a^{2}\right)^{2}}{2 \pi b^{3}(\operatorname{Det} G)^{1 / 4}} \frac{\operatorname{Det}(\mathbb{I}-X)^{3 / 4} \operatorname{Det}(\mathbb{I}+3 X)^{1 / 4}}{\operatorname{det}(1-X)^{3 / 2} \operatorname{det}(1+3 X)^{1 / 2}}
\end{aligned}
$$

If the quantity $\mathcal{R}$ equals 1 (see the comment below on this issue), this equation is exactly what is expected for the ratio of a flat static D25-brane action and a D23-brane action per unit volume in the presence of the $B$ field (2.14). In fact the DBI Lagrangian for a flat static Dp-brane is, [6],

$$
\mathcal{L}_{D B I}=\frac{1}{g_{s}(2 \pi)^{p}} \sqrt{\operatorname{Det}(1+2 \pi B)}
$$

where $g_{s}$ is the closed string coupling. Substituting (2.14) and taking the ratio the claim follows.

To end this section let us briefly discuss the generalization of the above results to lower dimensional lumps. As remarked at the end of section 2, every couple of transverse directions corresponding to an eigenvalue $B_{i}$ of the field $B$ can be treated in the same way as the 24-th and 25-th directions. One has simply to replace in the above formulas $B$ with $B_{i}$. The derivation of the above formulas for the case of $25-2 i$ dimensional lumps is straightforward.

\section{A comment}

Switching on a constant $B$ field on VSFT does not obstruct the possibility to find exact results. On the contrary, we have found that (matter) squeezed states representing tachyonic lumps are still solutions of the equations of motion, and that we can give compact explicit formulas for these solutions, much like in the $B=0$ case. These are still interpretable as 
(lower dimensional) D-branes. We have seen that the expected ratios for their tensions are reproduced, modulo the hypothesis that in (4.15, 4.16), the ratio $\mathcal{R}$ be equal to 1 . Let us discuss this briefly.

Let us recall that, as $B \rightarrow 0(a \rightarrow 0)$,

$$
x_{M N} \rightarrow X_{M N}^{\prime}
$$

where $X^{\prime}$ is the matrix used in 11] to define lower dimensional tachyonic lumps. It was shown there, numerically, that

$$
\frac{9 A^{4}}{2 \pi b^{3}} \frac{\operatorname{det}\left(1-X^{\prime}\right)^{3 / 2} \operatorname{det}\left(1+3 X^{\prime}\right)^{1 / 2}}{\operatorname{det}(1-X)^{3 / 2} \operatorname{det}(1+3 X)^{1 / 2}}
$$

is actually 1, and that this result does not seem to depend on the values taken by the parameter $b$. Now, (5.2) is exactly the limit of (4.16) when $a \rightarrow 0$. Therefore, looking at the structure of $\mathcal{X}$, it seems reasonable to assume that (4.16) is also 1 , at least as long as $a$ is not too large. In the limit $a \rightarrow \infty$ the exponent in the second line of (4.13) becomes illdefined and some kind of rescaling becomes necessary. However, before going deeply into this issue and the previos assumption, we believe that a better knowledge of the eigenvalues of $X$ is needed. This requires an ad hoc analysis, which will be done elsewhere along the lines of [20].

Note Added. After we had completed this work, there appeared a new paper by K.Okuyama, [26], who, using in particular the results of [20] and [27], was able to analytically prove that $(5.2)$ is exactly 1 . Using the same kind of arguments we have been able to prove analytically that in fact the ratio $\mathcal{R}$ in (4.16) is also 1 . The long details of this calculation will appear elsewhere, [28], together with new results on VSFT in the presence of a $B$ field.

\section{Acknowledgments}

We would like to thank Martin Schnabl for calling our attention on ref.[26]. This research was supported by the Italian MIUR under the program "Teoria dei Campi, Superstringhe e Gravità".

\section{A. Derivation of $(U \tilde{U})_{N M}^{\alpha \beta}$}

In this Appendix we derive eq.(3.20). This can be done starting both from the representation (3.12) and from (3.16). In the first case we need the following identities taken from the Appendix B of [11].

$$
\sum_{n \geq 1} W_{n} U_{n m}=W_{m}, \quad \sum_{n \geq 1} W_{n}^{*} W_{n}=2 V_{00}
$$

The numbers $W_{n}$ are defined via the equation

$$
V_{0 n}^{r s}=\frac{1}{3}\left(\omega^{s-r} W_{n}+\omega^{r-s} W_{n}^{*}\right)
$$


On the other hand we have

$$
\begin{array}{ll}
V_{0 n}^{r s}=\frac{i}{\sqrt{3}}\left(\omega^{r-s}-\omega^{s-r}\right) Z_{n}, & n \quad \text { odd } \\
V_{0 n}^{r s}=-\frac{1}{\sqrt{3}}\left(\omega^{r-s}+\omega^{s-r}\right) Z_{n}, & n \quad \text { even }
\end{array}
$$

This allows us to identify $W_{n}$ and $Z_{n}$ as follows:

$$
\begin{aligned}
& W_{n}=-i \sqrt{3} Z_{n}, \quad n \text { odd } \\
& W_{n}=-\sqrt{3} Z_{n}, \quad n \text { even }
\end{aligned}
$$

In particular, from the second equation in (A.1), we get

$$
\sum_{n \geq 1} Z_{n}^{2}=\frac{2}{3} V_{00}
$$

Next one has to consider $(\mathcal{U} \tilde{\mathcal{U}})_{N M}$ case by case according to the various possibilities for $N, M$. As a sample, let us consider $N=n$ odd and $M=m$ odd. Then

$$
(\mathcal{U} \tilde{U})_{n m}=\mathcal{U}_{n 0} \tilde{U}_{0 m}+\sum_{k \text { odd }} \mathcal{U}_{n k} \tilde{\mathcal{U}}_{k m}+\sum_{k \text { even }} \mathcal{U}_{n k} \tilde{\mathcal{U}}_{k m}
$$

Now we replace on the RHS the values extracted from eq.(3.12). After rearranging the terms we get

$$
\begin{aligned}
(\mathcal{U} \tilde{U})_{n m}= & G \delta_{n m}+\frac{b}{3} R \tilde{R} Z_{n} Z_{m}+R \tilde{R} Z_{n} Z_{m} \sum_{k \geq 1} Z_{k}^{2} \\
& -\frac{i}{\sqrt{3}} G \tilde{R} \sum_{k \geq 1} U_{n k} W_{k}^{*} Z_{m}+\frac{i}{\sqrt{3}} R G Z_{n} \sum_{k \geq 1} W_{k} U_{k m} \\
= & G \delta_{n m}\left(R G+G \tilde{R}+\frac{2}{3}\left(V_{00}+\frac{b}{2}\right) R \tilde{R}\right) Z_{n} Z_{m}
\end{aligned}
$$

where use has been made of (A.1) and (A.5). In the same way all other cases of the identity (3.20) can be proved.

Alternatively one can prove $(3.20)$ by means of the representation (3.16). The procedure is the same, but the matrix involved is $U^{\prime}$ instead of $U$. For this reason we need, instead of the second eq.A.1), the identity

$$
\sum_{n \geq 1} W_{n} U_{n m}^{\prime}=\frac{\frac{b}{2}-V_{00}}{\frac{b}{2}+V_{00}} W_{m}
$$

\section{References}

[1] E.Witten, Noncommutative Geometry and String Field Theory, Nucl.Phys. B268 (1986) 253.

[2] F.Sugino, Witten's Open String Field Theory in Constant B-field Background, JHEP 0003, (2000) 017 hep-th/9912254]. 
[3] T.Kawano and T.Takahashi, Open String Field Theory on Noncommutative Space, Prog. Theor. Phys. 104 (2000) 459 hep-th/9912274].

[4] E.Witten, Noncommutative Tachyons and String Field Theory, hep-th/0006071.

[5] M.Schnabl, String field theory at large B-field and noncommutative geometry, JHEP 0011, (2000) 031 hep-th/0010034.

[6] N.Seiberg and E.Witten, String Theory and Noncommutative Geometry, JHEP 9909, (1999) 032 hep-th/9908142].

[7] D.J.Gross and A.Jevicki, Operator Formulation of Interacting String Field Theory, Nucl.Phys. B283 (1987) 1.

[8] D.J.Gross and A.Jevicki, Operator Formulation of Interacting String Field Theory, 2, Nucl.Phys. B287 (1987) 225.

[9] V.A.Kostelecky and R.Potting, Analytical construction of a nonperturbative vacuum for the open bosonic string, Phys. Rev. D 63 (2001) 046007 hep-th/0008252].

[10] L.Rastelli, A.Sen and B.Zwiebach, String field theory around the tachyon vacuum, hep-th/0012251.

[11] L.Rastelli, A.Sen and B.Zwiebach, Classical solutions in string field theory around the tachyon vacuum, hep-th/0102112.

[12] L.Rastelli, A.Sen and B.Zwiebach, Half-strings, Projectors, and Multiple D-branes in Vacuum String Field Theory, JHEP 0111 (2001) 035 hep-th/0105058.

[13] D.Gaiotto, L.Rastelli, A.Sen and B.Zwiebach, Ghost Structure and Closed Strings in Vacuum String Field Theory, hep-th/0111129.

[14] H.Hata and T.Kawano, Open string states around a classical solution in vacuum string field theory, JHEP 0111 (2001) 038 hep-th/0108150.

[15] J.R.David, Excitations on wedge states and on the sliver, JHEP 0107 (2001) 024 hep-th/0105184.

[16] G.Moore and W.Taylor The singular geometry of the sliver, hep-th/0111069.

[17] K.Okuyama, Siegel Gauge in Vacuum String Field Theory, hep-th/0111087.

[18] K.Okuyama, Ghost Kinetic Operator of Vacuum String Field Theory, hep-th/0201015.

[19] L.Rastelli, A.Sen and B.Zwiebach, A note on a Proposal for the Tachyon State in Vacuum String Field Theory, hep-th/0111153.

[20] L.Rastelli, A.Sen and B.Zwiebach, Star Algebra Spectroscopy, hep-th/0111281.

[21] I.Kishimoto, Some properties of string field algebra, JHEP 0112 (2001) 007 hep-th/0110124.

[22] P.Mukhopadhyay, Oscillator representation of the BCFT construction of D-branes in vacuum string field theory, JHEP 0112 (2001) 025 hep-th/0110136].

[23] D.J.Gross and W.Taylor, Split string field theory. I JHEP 0108 (2001) 009 hep-th/0105059. D.J.Gross and W.Taylor, Split string field theory. II JHEP 0108 (2001) 010 hep-th/0106036 
[24] T.Kawano and K.Okuyama, Open String Fields as Matrices, JHEP 0106 (2001) 061 hep-th/0105129.

[25] N.Moeller, Some exact results on the matter star-product in the half-string formalism, hep-th/0110204.

[26] K.Okuyama, Ratio of Tensions from Vacuum String Field Theory, hep-th/0201136.

[27] H.Hata and S.Moriyama, Observables as Twist Anomaly in Vacuum String Field Theory, hep-th/0111034.

[28] L.Bonora, D.Mamone and M.Salizzoni, in preparation. 